\title{
Mobile robot applications grounded in deep learning theories: a review
}

\begin{abstract}
Mobile robots facing a diversity of open environments and performing a variety of tasks and interactions need explicit deliberation in order to fulfill their missions. Deliberation is meant to endow a mobile robot system with extended, more adaptable and robust functionalities, as well as reduce its deployment cost. The ambition of this survey is to present a global overview of deliberation functions in mobile robots and to discuss the state of the art in deep learning theories. And the present article focuses on the study of the intelligent navigation techniques, which are capable of navigating a mobile robot autonomously in static as well as dynamic environments.
\end{abstract}

Keywords: mobile robot survey, deep learning, technological development, predictive model, visual navigation, deep convolution neural networks
Volume 3 Issue 5 - 2017

\author{
Wei Zheng,' Hong-bin Wang,' Zhi Ming \\ Zhang' \\ 'Institute of Electric Engineering, Yanshan University, China \\ ${ }^{2}$ China National Heavy Machinery research institute, China
}

Correspondence: Wei Zheng, Institute of Electric Engineering,
Yanshan University, China, Tel +86-13722559060,

Email xiaoxiong19871110@163.com

Received: August 29, 2017 | Published: November 21, 2017

\section{Introduction}

The mobile robot technology occupies a wide space in surgical complex procedure, in particular the da Vinci System. ${ }^{1}$ In addition, technological development is increasingly incorporated in our everyday lives. Several robots applications in education are mainly concerned with supporting the teaching of subjects directly related to the mobile robot field, such as mobile robot programming and construction. ${ }^{2}$ Basically, the mobile robot navigation has been done by the Deterministic algorithm and Nondeterministic (Stochastic) algorithm. Nowadays, the hybridization of both the algorithms called as an Evolutionary algorithm is being used to solve the mobile robot navigation problem. The applications of the autonomous mobile robot in many fields such as industry, space, defence and transportation, and other social sectors are growing day by day. ${ }^{3}$ However, exploring a wider range of possible applications of flexible robots has the potential to engage young people with a wider range of interests, as illustrated with experiences in subjects related to music and art. The main contribution of this survey is to systematically review relevant educational mobile robot experiences with theoretical support on tertiary education.

\section{Methods}

Most of the literatures on deep learning theories are focused on path planning However, path planning alone is not sufficient for achieving deliberate action. ${ }^{4}$ Acting is accomplished by exerting controlled forces on the environment. It is implemented at the lowest level by online processing of streams of signals, from sensors to actuators. These streams achieve feedback loops robust to the discrepancies between the world and its models. Planning, on the other hand, implements a mapping of its input, a predictive model and a problem, into an output plan expressed in some representation. ${ }^{5}$ Because of the limitation of predictive models and complexity constraints, planning has to admit several simplifying assumptions. As a result, a synthesized plan provides a projected course of action in some abstract representation. It is useful but rarely sufficient for acting deliberately.

\section{Results}

The deep convolution neural networks have the ability to accommodate certain degree of transformation, deformation and illumination variation. It also has a light computational load for scanning the entire image to detect interested objects. Therefore, deep convolution neural network could be a good solution to meet the above challenges. It is based on unsupervised and supervised feature learning and has been widely used in many applications, where its structure is designed with multiply network layers to simulate learning process of human beings.

\section{Discussion}

The feature learning algorithms, especially convolutional neural networks, have been widely used in many areas, i.e., computer vision and speech recognition. ${ }^{6,7}$ However, it is seldom used in mobile visual navigation. Due to the advantages of the method, it is used for door detection in indoor environments for mobile robot navigation. This process contains two steps. Firstly, a judgment is made to check whether a door exists in the image. Secondly, it can locate the door to control the robot how to act, for example moving through the door. In the experiments, people use the images with five poses of the door to train the networks. ${ }^{6}$ The objectives are to make the mobile robot know the rough location of door, and then make decision of how to move to the door and go through it.

\section{Conclusion}

In this survey, the utility of the deep reinforcement learning framework for the mobile robot exploration is discussed. In particular, we considered the deep learning applications of robots as auxiliary tools in visual navigation. The deep reinforcement learning model extends the cognitive ability of mobile robots for more complicated indoor environments in an efficient online-learning process continuously. As a result, this work extends a previous systematic review ${ }^{7}$ by taking into account a different educational level. Moreover, the current systematic review expands the previous one by paying attention to the learning theories support for practical activities with robots.

\section{Acknowledgments}

This research was financially supported by National Natural Science Foundation of China (No. 61473248, No. 6177333); Natural Science Foundation of Hebei Province (No. F2016203496). 


\section{Conflict of interest}

The authors declare that there is not conflict of interests regarding the publication of this paper.

\section{References}

1. Spolaôr, Newton, FBV Benitti. Robotics applications grounded in learning theories on tertiary education: A systematic review. Computers \& Education. 2017;112:97-107.

2. Charalampous K, Kostavelis I, Gasteratos A. Recent trends in social aware robot navigation: A survey. Robotics \& Autonomous Systems. 2017;93:85104.
3. Anish Pandey, Shalini Pandey, Dayal R, et al. Mobile Robot Navigation and Obstacle Avoidance Techniques: A Review. International Robotics \& Automation Journal. 2017;2(3):1-12.

4. Rusk N. Deep learning. Nature Methods. 2016;13(1):35-35.

5. Ingrand, Félix, M Ghallab. Deliberation for autonomous robots: A survey. Artificial Intelligence. 2014;247:10-44.

6. Sun Yi, Wang X, Tang X. Hybrid Deep Learning for Face Verification. IEEE Transactions on Pattern Analysis \& Machine Intelligence. 2016;38(10):1997-2009.

7. Guo YM, Liu Y, Oerlemans A, et al. Deep learning for visual understanding. Neurocomputing. 2016;187(C):27-48. 